Bundesgesundheitsbl 2017 · 60:1180-1183

DOI 10.1007/s00103-017-2613-y

๑) Springer-Verlag GmbH Deutschland 2017
Empfehlung des Umweltbundesamtes

\section{Empfehlung zu erforderlichen Untersuchungen auf Pseudomonas aeruginosa, zur Risikoeinschätzung und zu Maßnahmen beim Nachweis im Trinkwasser}

\section{Empfehlung des Umweltbundesamtes nach Anhörung der Trinkwasserkommission}

\section{Anlass}

$\$ 5$ TrinkwV 2001 [1] regelt die Anforderungen an die Trinkwasserqualität aus mikro-biologischer Sicht. Nach Abs. 1 dürfen im Trinkwasser Krankheitserreger im Sinne des $₫ 2$ Abs. 1 des Infektionsschutzgesetzes (IfSG) [2], die durch Wasser übertragen werden können, nicht in Konzentrationen enthalten sein, die eine Schädigung der menschlichen Gesundheit besorgen lassen.

In $\$ 5$ Abs. 4 TrinkwV 2001 ist geregelt, dass die Konzentrationen von Mikroorganismen so niedrig gehalten werden sollen, wie dies nach den allgemein anerkannten Regeln der Technik mit vertretbarem Aufwand unter Berücksichtigung der Umstände des Einzelfalls möglich ist. Dies trifft auf Mikroorganismen zu, die das Trinkwasser verunreinigen oder seine Beschaffenheit nachhaltig beeinflussen können. Hierzu gehört auch Pseudomonas aeruginosa ( $P$. aeruginosa), der als wichtiger fakultativ-pathogener, durch Wasser übertragbarer Krankheitserreger gilt.

Umfang und Häufigkeit der Überwachung durch den Betreiber der Wasserversorgungsanlage und der amtlichen Überwachung durch die Gesundheitsämter als die zuständigen Gesundheitsbehörden regelt die Trinkwasserverordnung. Im Gegensatz zu Escherichia coli und Entero- kokken ist $P$. aeruginosa kein Parameter, der regelmäßig in die Untersuchung von Trinkwasserproben einbezogen wird.

Das zuständige Gesundheitsamt kann nach $\$ 20$ TrinkwV 2001 eine anlassbezogene Untersuchung auf $P$. aeruginosa anordnen um festzustellen, ob die Anforderungen des $\$ 5$ Abs. 1 TrinkwV 2001 erfüllt sind und eine mikrobiologische Qualität des Trinkwassers gewährleistet ist, die eine Schädigung der menschlichen Gesundheit durch Verwendung des Wassers für den menschlichen Gebrauch nicht besorgen lässt. Eine derartige Anordnung erfordert eine auf die konkrete Situation angepasste Begründung zur Durchführung dieser Untersuchungen. Erfahrungen zeigen, dass insbesondere im Zusammenhang mit Baumaßnahmen (Neubau und bauliche Veränderungen) im Leitungsnetz der öffentlichen Trinkwasserversorgung und in der Trinkwasser-Installation $P$. aeruginosa eingetragen werden kann und daher eine Untersuchung des Trinkwassers auf $P$. aeruginosa einen Beitrag zur hygienischen Sicherheit leistet. Die TWK sieht es als erforderlich an zu beschreiben, wann auf das Vorkommen von $P$. aeruginosa im Trinkwasser entsprechend dem Besorgnisgrundsatzes nach $\$ 37$ IfSG zu untersuchen ist und welche Konzentration nicht im Trinkwasser enthalten sein darf.
Die Empfehlung ersetzt die 2002 herausgegebene Empfehlung [3] sowie in Bezug auf $P$. aeruginosa die Empfehlung von 2005 [4] und gibt eine hygienischmedizinische Bewertung. Adressaten sind Gesundheitsämter, Wasserversorger und Unternehmer oder sonstige Inhaber (UsI) einer Trinkwasser-Installation sowie Trinkwasseruntersuchungsstellen.

\section{Vorkommen}

$P$. aeruginosa ist ein ubiquitär verbreitetes Umweltbakterium, das als natürlicher Bewohner im feuchten Milieu in geringen Konzentrationen vorkommt (assoziiert in Erde und Wasser). P. aeruginosa zeichnet sich durch Eigenschaften wie geringe Nährstoffansprüche, Wachstum in einem breiten Temperaturbereich sowie die Fähigkeit zur Biofilmbildung aus und ist in der Lage, sowohl in wasserführenden Systemen als auch in destilliertem Wasser zu überleben und sich zu vermehren [5]. Der Eintrag in das Trinkwassersystem kann über kontaminierte Materialien bei Bauarbeiten am Leitungsnetz oder bei Arbeiten an bestehenden bzw. neu errichteten Trinkwasser-Installationen erfolgen [6]. Als Folge eines Eintrages ist die Besiedlung verschiedenster Materialklassen wie Edelstahl, Kunststoffe, Weich-PVC oder Dichtmaterialien möglich, wobei neue 
Materialien ohne bestehenden Biofilm stärker besiedelt werden als Oberflächen mit einem vorhandenen Trinkwasser-Biofilm. Eine Vermehrung ist nur dann gegeben, wenn eine Nährstoffquelle vorliegt, z.B. auf der Oberfläche von Materialien ohne Eignung entsprechend DVGW W $270[7,8]$.

\section{Gesundheitliche Aspekte}

P. aeruginosa gilt als fakultativ pathogener Krankheitserreger. Insbesondere bei Personen mit prädisponierenden Faktoren besteht die Besorgnis einer Infektion; dazu zählen invasive Fremdkörpersysteme wie Harnwegskatheter, Venenkatheter, Beatmungstuben, aber auch offene akute und chronische Wunden, Verbrennungen sowie bestimmte Grunderkrankungen wie Mukoviszidose. Daher besitzt $P$. aeruginosa als Erreger nosokomialer Infektionen wie Harnwegsinfektionen, Pneumonien oder Septikämien große Bedeutung in medizinischen Einrichtungen. Trinkwasserassoziierte $P$. aeruginosa-Infektionen in medizinischen Einrichtungen sind gut dokumentiert und konnten durch Sanierung bzw. endständige Filter unter Kontrolle gebracht werden $[9,10]$.

Aber auch bei Personen ohne prädisponierende Faktoren kann $P$. aeruginosa zu einer Infektion führen, das Spektrum der Erkrankungen bei immunkompetenten Personen schließt Wundinfektionen, Ohrinfektionen (Otitis externa), Bindehautentzündungen (Keratokonjunktivitis) und Haarbalginfektionen (Folliculitis) ein.

Der Haupteintragspfad von $P$. aeruginosa über kontaminiertes Wasser ist der Kontakt mit verletzter Haut. Reinigung und Aufbewahrung von Kontaktlinsen in kontaminiertem Wasser sind weitere Infektionsquellen [11].

$P$. aeruginosa gilt zudem als einer der häufigsten Erreger von chronischen Wundinfektionen, die bei älteren Personen wie auch Personen mit Diabetes eine wichtige epidemiologische Bedeutung haben [12].

Aufgrund der großen Anpassungsfähigkeit und Antibiotikaresistenz des Erregers sind nosokomiale $P$. aerugino$s a$-Infektionen und chronische Wundinfektionen nur schwer behandelbar und können unter Umständen zum Tod führen. Die Heilungschancen sind von verschiedenen Faktoren, wie dem betroffenen Organsystem, der Virulenz und Resistenz des Stammes, dem Ausmaß der Infektion und dem individuellen Gesundheitszustand des Erkrankten, abhängig [13].

Fazit: Als Erreger sporadischer Infektionen und aufgrund der hohen Antibiotikaresistenz sind durch $P$. aerugino$s a$ ausgelöste Infektionen aus Sicht der öffentlichen Gesundheit so bedeutsam, dass alle Möglichkeiten der Verhütung, einschließlich der Prävention wasserassoziierter Infektionen, ergriffen werden müssen.

\section{Trinkwasserhygienische Aspekte}

\subsection{Neubau und Baumaßnahmen im Leitungsnetz und in der Trinkwasser-Installation}

Im Wasser zeichnet sich $P$. aeruginosa durch eine hohe Tenazität und lange Persistenz durch Biofilmbildung aus. $P$. aeruginosa ist somit ein geeigneter Indikator für hygienisch-technische Mängel in Trinkwasser-Installationen und Verteilungssystemen $[6,7]$.

Mögliche Eintrags- und Animpfquellen sind der Einbau kontaminierter Bauteile (z.B. ein kontaminierter Wasserzähler; [8]) sowie ein Schmutzeintrag aufgrund fehlerhafter Lagerung von Bauteilen während einer Baumaßnahme.

Begünstigende Faktoren sind eine unsachgemäße Inbetriebnahme (z. B. Stagnation vor Nutzungsbeginn), eine erhöhte Temperatur im Kaltwasserbereich sowie eine materialbedingte Biofilmbildung (siehe technisches Regelwerk).

Zur Absicherung einer einwandfreien Trinkwasserqualität wird daher empfohlen, die Trinkwasserbeschaffenheit im Leitungsnetz nach Neubau bzw. nach der Durchführung von Baumaßnahmen (z. B. Erneuerung von Leitungen und Bauteilen, Baumaßnahmen mit Leitungsöffnung) durch Untersuchung auf $P$. aeruginosa zu kontrollieren. Diese Untersuchung soll vor Einbindung in die Versorgung erfolgen.

Darüber hinaus wird empfohlen, nach einem Neubau von Trinkwasser-Installati- onen in Krankenhäusern, Alten- und Pflegeheimen, Zahnarztpraxen sowie Arztpraxen und Praxen sonstiger Heilberufe, in denen invasive Eingriffe vorgenommen werden, sowie in Kindereinrichtungen eine Untersuchung auf $P$. aeruginosa durchzuführen. Sinnvoll sind auch Untersuchungen nach Umbau- oder Sanierungsarbeiten.

\subsection{Einrichtungen nach § 19 Abs. 7 TrinkwV 2001}

Nach $\$ 19$ Abs. 7 TrinkwV 2001 muss das Gesundheitsamt Trinkwasser-Installationen, aus denen Trinkwasser im Rahmen einer öffentlichen Tätigkeit zur Verfügung gestellt wird, in ein stichprobenartiges Überwachungsprogramm einbinden und dabei mindestens auf die Parameter untersuchen oder untersuchen lassen, die sich in der Trinkwasser-Installation nachteilig verändern können. Für bestimmte Einrichtungen ist daher $P$. aeruginosa in die regelmäßige Überwachung einzubeziehen. Dazu zählen aus Gründen der Gesundheitsvorsorge in Anlehnung an $\$ \$ 23$, 33 und 36 IfSG folgende Einrichtungen:

a) Krankenhäuser und Pflegeeinrichtungen (Altenpflegeheime, Pflegeheime),

b) Einrichtungen für ambulantes Operieren, Dialyseeinrichtungen, Tageskliniken, Entbindungseinrichtungen, Einrichtungen zur Rehabilitation,

c) Kindertagesstätten.

Für Einrichtungen der Kategorie a) bis c) werden mindestens jährliche Untersuchungen empfohlen.

In den übrigen öffentlichen Einrichtungen der Kategorie d) bis h) kann eine Untersuchung auf. P. aeruginosa nach Risikoeinschätzung durch das Gesundheitsamt veranlasst werden. Hierzu zählen:

d) Schulen

e) Hotels, Jugendherbergen

f) Sonstige Ausbildungseinrichtungen, Heime, Ferienlager u. ä. Einrichtungen

g) Sportstätten

h) weitere Gemeinschaftsunterkünfte entsprechend $\$ 36$ IfSG, Einrichtungen nach $₫ 1$ Abs. 1 bis 5 des Heimgesetzes, Betreuungs- oder Versorgungseinrichtungen, die mit einer der in den Nummern 1 und 2 genann- 


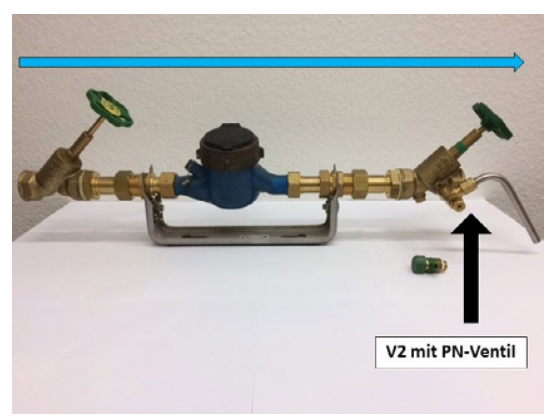

Abb. $1 \Delta$ Wasserzähleranlage mit Entnahmevorrichtung für mikrobiologische und chemische Wasserproben am Ventil V2 (Entleerung/Kundenseite)

ten Einrichtungen vergleichbar sind, Obdachlosenunterkünfte, Gemeinschaftsunterkünfte für Asylbewerber, Spätaussiedler und Flüchtlinge, sonstige Massenunterkünfte und Justizvollzugsanstalten.

Die Häufigkeit sollte in Abstimmung mit dem Gesundheitsamt in Abhängigkeit von der Art der Einrichtung und dem tatsächlichen Gefährdungspotential aufgrund des Zustandes der Trinkwasser-Installation festgelegt werden.

Für Zahnarztpraxen sowie Arztpraxen und Praxen sonstiger Heilberufe, in denen invasive Eingriffe vorgenommen werden, sind insbesondere die Empfehlungen der Kommission für Krankenhaushygiene und Infektionsprävention des Robert Koch-Institutes zu beachten.

\section{Probennahme im Leitungsnetz und in der Trinkwasser-Installation}

Die Probennahmestellen sind nach DIN EN ISO 19458:2006 [14] auszuführen (gut zugänglich, abflammbar).

\subsection{Probennahme im Leitungsnetz}

Die Entnahme von mikrobiologischen Wasserproben im Leitungsnetz erfolgt nach DIN EN ISO 19458:2006 Tab. 1 Zweck a). Zur Feststellung der Trinkwasserbeschaffenheit im Leitungsnetz haben sich Probennahmestellen in Anlagen des Wasserversorgers (Behälter, Druckstationen usw.) oder an der Übergabestelle in die Trinkwasser-Installation bewährt. Von Beprobungen an Hydranten ist ab- zusehen, da diese u. U. mikrobiologische Befunde aufweisen können, die lediglich auf eine Verunreinigung der Probennahmevorrichtung zurückzuführen sind und nicht mit der Trinkwasserbeschaffenheit im Leitungsnetz korrelieren.

An der Übergabestelle kann die Probe mittels eines geeigneten Probennahmehahns an Hausanschlussleitungen nach dem Wasserzähler entnommen werden. Wenn keine zusätzlich installierte Entnahmearmatur in bzw. an der Wasserzähleranlage vorhanden ist, die gemäß DIN EN ISO 19458:2006 für mikrobiologische Entnahmen geeignet ist, bietet das Ventil V2 (Kundenseite) am Wasserzähler (Übergabestelle Wasserversorger - Trinkwasser-Installation) diese Möglichkeit. Dort kann zur fachgerechten Probennahme ein abflammbares Probenentnahmeventil (PN-Ventil) angebracht werden (• Abb. 1). Für die Untersuchung an neu errichteten Rohrleitungen wird der Einsatz eines Flansches mit Probennahmehahn am Rohrleitungsende empfohlen.

\subsection{Probennahme in der Trinkwasser-Installation an peripheren Stellen}

Die Probennahme erfolgt an peripheren Stellen in der Trinkwasser-Installation nach DIN EN ISO 19458:2006 Tab. 1 Zweck b). Die Festlegung, wo die Probennahmen durchgeführt werden sollen, orientiert sich an der konkreten Fragestellung, der Größe und der Art der Einrichtung mit dem Ziel, eine systemische Kontamination der Trinkwasser-Installation zu erkennen.

In medizinischen Einrichtungen erfolgt die Probennahme in Hochrisikoeinrichtungen wie Intensivtherapiestationen, hämatoonkologische Stationen, neonatologische Stationen (zusätzlich nach DIN EN ISO 19458:2006 Tab. 1 Zweck c).

\section{Nachweis und Bewertung von P. aeruginosa}

Der Nachweis von P. aeruginosa in Trinkwasserproben erfolgt mittels Membranfiltrationsverfahren nach DIN EN ISO 16266 bzw. Alternativverfahren (siehe UBA-Liste) $[15,16]$. P. aeruginosa kann jedoch auch als Nebenbefund bei anderen Trinkwasseruntersuchungen festgestellt werden, z.B. bei der Untersuchung auf Escherichia coli und coliforme Bakterien mittels Referenzverfahren der Trinkwasserverordnung oder bei der Untersuchung auf Legionellen. Der Nachweis ist immer im Untersuchungsbericht anzugeben.

Nach $\$ 15$ Abs. 4 TrinkwV 2001 dürfen Probennahmen und Untersuchungen des Trinkwassers nur von solchen Untersuchungsstellen durchgeführt werden, welche die dort gelisteten Anforderungen erfüllen, durch eine nationale Akkreditierungsstelle eines Mitgliedstaates der Europäischen Union für Trinkwasseruntersuchungen akkreditiert und von der zuständigen obersten Landesbehörde oder einer von ihr benannten Stelle dafür zugelassen sind.

P. aeruginosa soll in $100 \mathrm{ml}$ nicht nachweisbar sein $(<1 \mathrm{KBE} / 100 \mathrm{ml})$.

\section{Empfehlungen zum Vorgehen beim Nachweis von $P$. aeruginosa in Trinkwasserproben}

\subsection{Nachweis im Zusammenhang mit einer Baumaßnahme}

Ist es als Folge von Baumaßnahmen zu einer Kontamination mit $P$. aeruginosa gekommen, sollte als erste Maßnahme eine Spülung durchgeführt werden, um ggf. vorhandene Verunreinigungen auszutragen. Hinweise zur Durchführung der Spülung geben die DVGW-Arbeitsblätter W 291 [17] für das Leitungsnetz und W 557

[18] für die Trinkwasser-Installation.

Ist nach der Spülung $P$. aeruginosa weiterhin nachweisbar, ist von einer Oberflächenkontamination auszugehen. Um eine Beseitigung der Oberflächenkontamination zu erreichen, ist eine chemische oder thermische Anlagendesinfektion entsprechend DVGW-Regelwerk (W 291, W 557) durchzuführen. Mit Ausnahme von Dichtungsmaterialien wird bei allen Materialien eine vollständige Beseitigung der Oberflächenkontamination erreicht, sofern die Oberflächen mit dem Desinfektionsmittel in Kontakt kommen und die Einwirkzeit ausreichend lang ist. Für Dichtungsmaterialien ist meist nur eine unvollständige Beseitigung der Oberflächenkontamination möglich. 
Ist zur Beseitigung einer Oberflächenkontamination keine Anlagendesinfektion umsetzbar, sollte eine kontinuierliche Desinfektion des Trinkwassers zur Aufrechterhaltung einer Desinfektionsmittelrestkonzentration gemäß TrinkwV 2001 über mindestens vier Wochen durchgeführt werden. Hierbei ist sicherzustellen, dass im kontaminierten Bereich freies Desinfektionsmittel vorhanden ist und alle Innenoberflächen mit dem Desinfektionsmittel in Kontakt kommen. $\mathrm{Zu}$ beachten ist, dass die Wirkung von der vorliegenden Desinfektionsmittelkonzentration abhängig ist. Um zu überprüfen, ob durch die Desinfektionsmaßnahme eine nachhaltige Beseitigung erreicht wurde, ist die Desinfektionsmittelkonzentration schrittweise abzusenken und dies mit einem entsprechenden Untersuchungsprogramm zu begleiten.

Sollten während oder nach der Verringerung der Desinfektionsmittelkonzentration bzw. der Abschaltung weiterhin Befunde auftreten, ist im Rahmen eines Messprogramms die Kontaminationsstelle zu identifizieren und das entsprechende Anlagenteil auszutauschen.

Der Erfolg der durchgeführten Maßnahmen ist durch entsprechende Untersuchungen zu kontrollieren.

\subsection{Nachweis im Rahmen der Überwachung der Trinkwasser- Installation nach § 19 Abs. 7 TrinkwV 2001}

Wird bei der Untersuchung in der Trinkwasser-Installation P. aeruginosa nachgewiesen, ist die Kontaminationsstelle zu identifizieren und zu beseitigen. Zunächst sollte durch Untersuchungen an der Übergabestelle geprüft werden, ob die Kontamination bereits im Leitungsnetz vorliegt (z. B. Baumaßnahmen) oder auf die Trinkwasser-Installation zurückzuführen ist. Im ersten Fall hat das Gesundheitsamt unverzüglich mit dem Wasserversorger Kontakt aufzunehmen und entsprechende Maßnahmen zu veranlassen.

Ist die Kontamination auf die Trinkwasser-Installation zurückzuführen, werden die unter 7.1 beschriebenen Maßnahmen zur Beseitigung der Kontamination empfohlen (siehe auch DVGW-Arbeitsblatt W 556 [19]). Der Erfolg der durch- geführten Maßnahmen ist durch entsprechende Untersuchungen zu kontrollieren. Eine nachhaltige Sanierung in der Trinkwasser-Installation ist erreicht, wenn ohne Zugabe von Desinfektionsmitteln auch nach 12 Wochen $P$. aeruginosa in $100 \mathrm{ml}$ nicht nachweisbar ist [19].

Eine dauerhafte chemische Desinfektion des Trinkwassers in der TrinkwasserInstallation widerspricht dem Minimierungsgebot nach $\$ 6$ TrinkwV 2001 und ersetzt nicht die ggf. notwendigen Sanierungsmaßnahmen.

\subsection{Vermeidung vor Sanierung}

Die Sanierung von Trinkwasser-Installationen oder Leitungsnetzen nach Kontamination mit $P$. aeruginosa ist erfahrungsgemäß sehr aufwändig und in Einzelfällen nur durch kompletten Austausch der kontaminierten Bauteile möglich. Die Vermeidung von Kontaminationen ist daher deutlich effektiver und effizienter. In Frage kommen technische, organisatorische und personelle Maßnahmen, z. B. die konsequente Nutzung von Endkappen auf Rohrleitungen und Armaturen, die angemessene Organisation von Baustellen sowie die Sicherstellung adäquater Hygieneunterweisungen (siehe technisches Regelwerk).

\section{Literatur}

1. Trinkwasserverordnung in der Fassung der Bekanntmachung vom 10. März 2016 (BGBI. I S. 459), die durch Artikel 4 Absatz 21 des Gesetzes vom 18. Juli 2016 (BGBI. I S. 1666) geändert worden ist

2. Infektionsschutzgesetz vom 20. Juli 2000 (BGBI. IS. 1045), das zuletzt durch Artikel 4 Absatz 20 des Gesetzes vom 18. Juli 2016 (BGBI. I S. 1666) geändert worden ist

3. Umweltbundesamt, Trinkwasserkommission (2002) Empfehlung der Trinkwasserkommission zur Risikoeinschätzung, zum Vorkommen und zu Maßnahmen beim Nachweis von Pseudomonas aeruginosa in Trinkwassersystemen. Bundesgesundheitsbl Gesundheitsforsch Gesundheitsschutz 45:187-188

4. Umweltbundesamt, Trinkwasserkommission (2005) Hygienisch-mikrobiologische Untersuchung im Kaltwasser von Wasserversorgungsanlagen nach § 3 Nr. 2 Buchstabe c TrinkwV 2001, aus denen Wasser für die Öffentlichkeit im Sinne des § 18 Abs. 1 TrinkwV 2001 bereitgestellt wird. Bundesgesundheitsbl Gesundheitsforsch Gesundheitsschutz 49:693-696

5. Botzenhart K, Döring G (1993) Ecology and Epidemiology of Pseudomonas aeruginosa. In: Campa
M, Bendinelli M, Friedman H (Hrsg) Pseudomonas aeruginosa as an opportunistic pathogen. Springer, New York, S 1-18

6. Wingender J, Hambsch B, Schneider S (2009) Mikrobiologisch-hygienische Aspekte des Vorkommens von Pseudomonas aeruginosa im Trinkwasser. DVGW energie:60-66

7. Hambsch B, Hügler M, Korth A, Petzoldt H (2016) Pseudomonas aeruginosa in Trinkwassersystemen - Wachstumsansprüche und nachhaltige Gegenmaßnahmen. In: Veröffentlichungen aus dem DVGW-Technologiezentrum Wasser Band 73: Pseudomonas aeruginosa in Trinkwassersystemen. TZW: DVGW-Technologiezentrum Wasser, Karlsruhe, S. 1-100

8. Hambsch B, Hügler M, Schönthal M, Kempf T, Maier M (2016) Einfluss von Wasserzählern auf die mikrobiologische Beschaffenheit der nachgeschalteten Trinkwasser-Installation. In: Veröffentlichungen aus dem DVGW-Technologiezentrum Wasser Band 73: Pseudomonas aeruginosa in Trinkwassersystemen. TZW: DVGW-Technologiezentrum Wasser, Karlsruhe, S. 101-151

9. Exner M, Nissing W, Behringer K, Engelhart $S$, Pleischl S, Koch C, Trautmann M, Kramer A, Walger P, Martiny H, Jatzwauk L (2016) Gesundheitliche Bedeutung, Prävention und Kontrolle Wasserassoziierter Pseudomonas aeruginosa-Infektionen. Hyg Med Supplement 2:1-32

10. Kanamori H, Weber D, Rutala W (2016) Healthcare outbreaks associated with a water reservoir and infection prevention strategies. Clin Infect Dis 62(11):1423-1435

11. Dyavaiah M, Phaniendra A, Sudharshan S (2015) Microbial Keratitis in Contact Lens Wearers. Jsm Ophthalmol 3(3):1036-1047

12. Gjødsbøl K, Christensen J, Karlsmark T, Jørgensen B, Klein B, Krogfelt K (2006) Multiple bacterial species reside in chronic wounds: a longitudinal study. Int Wound J 3(3):225-231

13. Exner M, Koch C, Gebel J (2011) Relevante wasserassoziierte Krankheitserreger sowie Indikatoren und deren hygienisch-medizinische Bedeutung. Umweltmedizin Forsch Prax 16(3):141-156

14. DIN EN ISO 19458:2006 Wasserbeschaffenheit - Probenahme für mikrobiologische Untersuchungen. Beuth, Berlin

15. DIN EN ISO 16266:2008 Wasserbeschaffenheit Nachweis und Zählung von Pseudomonas aeruginosa-Membranfiltrationsverfahren. Beuth, Berlin

16. Umweltbundesamt (2015) Liste alternativer mikrobiologischer Nachweisverfahren gemäß § 15 Absatz 1 TrinkwV 2001, 3. Änderungsmitteilung. https://www.umweltbundesamt.de/dokument/ liste-alternativer-mikrobiologischer. Zugegriffen: 22. Juni 2017

17. DVGW (2000) Arbeitsblatt W 291: Reinigung und Desinfektion von Wasserverteilungsanlagen

18. DVGW (2012) Arbeitsblatt W 557: Reinigung und Desinfektion von Trinkwasser-Installationen

19. DVGW (2015) Arbeitsblatt W 556: Hygienischmikrobielle Auffälligkeiten in Trinkwasser-Installationen; Methodik und Maßnahmen zu deren Behebung 